\title{
Evidence for a Mass Outflow from Our Galactic Center
}

\author{
Casey Law \\ Astronomical Institute "Anton Pannekoek", \\ University of Amsterdam, \\ Kruislaan 403, Amsterdam, 1098 SJ, Netherlands \\ email: claw@science.uva.nl
}

\begin{abstract}
We discuss the nature of the Galactic center lobe (GCL), a degree-tall, loop-like structure apparently erupting from the central few hundred parsecs of our Galaxy. Although its coincidence with the Galactic center has inspired diverse models for its origin, the observational evidence connecting this structure to the GC region has been thin. We describe a multiwavelength observing campaign with the VLA, GBT, Spitzer, and other telescopes that finds compelling evidence that the structure is likely formed by a mass outflow from the central tens of parsecs of our Galaxy. The size and mass of the putative outflow is consistent with that expected from the observed supernova rate and gas pressure in the GC region. If the GCL is a mass outflow, its relative proximity offers a unique opportunity for studying these structures in unprecedented detail.
\end{abstract}

Keywords. Galaxy: center - ISM: jets and outflows - galaxies: starburst

\section{Introduction}

Dynamical processes make galactic nuclei host to massive black holes, stellar clusters, and dense molecular gas. Energetic jets and bursts of star formation are associated with massive outflows of gas and dust out of galaxies (e.g., NGC 3079; Cecil et al. 2001). Understanding the effect of these outflows impacts upon a wide range of topics, from galaxy evolution to the enrichment of the intergalactic medium.

While massive outflows are typically seen in more massive and active galaxies, our own Galactic center (GC) may host mass outflows, judging from the presence of several unusual properties. Massive stellar clusters $\left(M_{\mathrm{cl}} \sim 10^{4} \mathrm{M}_{\odot}\right)$ have been found there with properties similar to those seen in starburst galaxies (Figer et al. 1999). The ionized gas excitation conditions are similar to that seen in minor starbursts (Rodríguez-Fernández \& Martín-Pintado 2004). The center of the Galaxy is known to have a massive $(\sim 4 \times$ $10^{6} \mathrm{M}_{\odot}$ ) black hole, such as found in active galaxies (Ghez et al. 2005). If the GC is host to an outflow, it would provide us with the closest view of this phenomenon, helping us understand them.

One possible signature of an outflow from our GC is the Galactic center lobe (GCL). The GCL was first discovered in radio continuum observations (Sofue \& Handa 1984). As shown in Figure 1, the GCL is a $\sim 1^{\circ}$ tall shell that spans the central $\sim 100$ pc of the GC region. Based on the coincidence of the shell with the GC and its morphology, it was suggested that the GCL is a sign of a mass outflow from the GC region. However, these ideas were founded mostly on morphology; the early observations allowed for the possibility that the east and west sides were unrelated to each other (Uchida et al. 1994). Bland-Hawthorn \& Cohen (2003) resurrected the idea that the GCL is caused by a mass outflow by noting the presence of dust emission surrounding the structure in MSX $8 \mu \mathrm{m}$ data. 

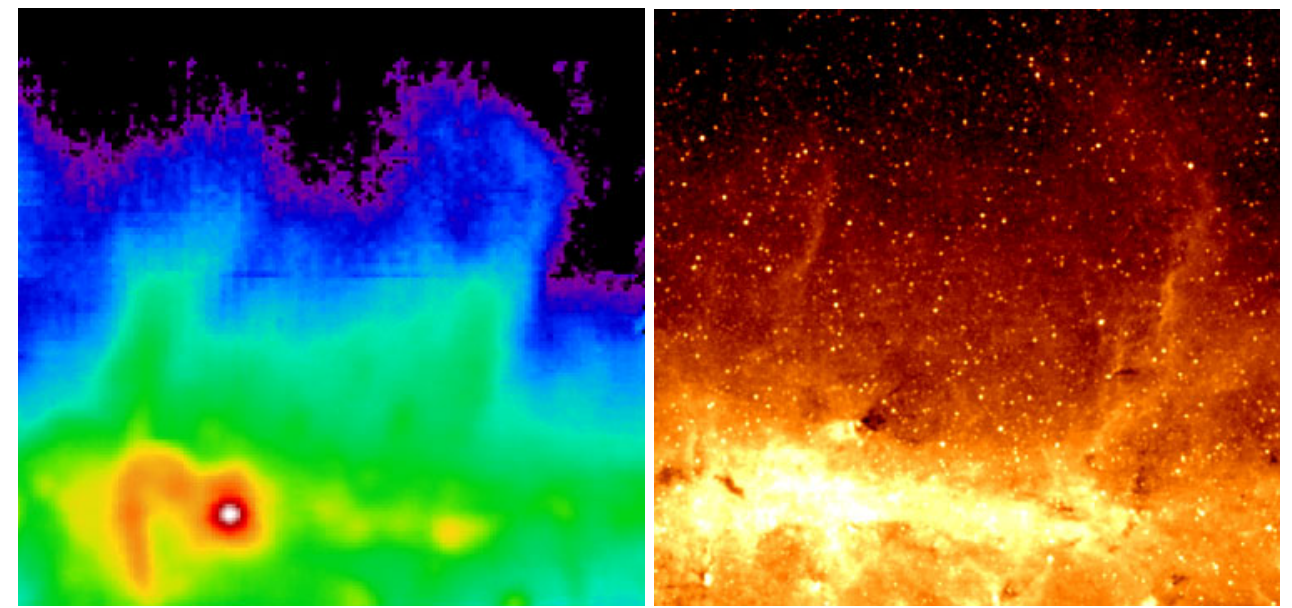

Figure 1. Left: Green Bank Telescope (GBT) $6 \mathrm{~cm}$ radio continuum image of the central two degrees of the GC region. The east and west sides of the GCL are seen here. Right: Midcourse Space Experiment (MSX) $8 \mu \mathrm{m}$ continuum image of the same region.

To address earlier questions about the nature of the GCL and quantify its physical conditions, we conducted a series of observations. This work summarizes the results of this multiwavelength study of the GCL and presents evidence that it is a signature of a GC mass outflow.

\section{New Results}

We have collected and compared observations of the GCL by conducting new observations and searching public data archives. This study has revealed new aspects of the structure of the GCL. Here we summarize the available observations of the GCL; a more complete description will be given elsewhere (Law et al. 2008).

\subsection{GBT Radio Continuum at 3.6, 6 and $20 \mathrm{~cm}$}

The radio continuum of the central few degrees of the GC region was surveyed at 3.6, 6 , and $20 \mathrm{~cm}$ wavelengths with the GBT. These observations resolved the radio continuum shell of the GCL that was first noted by Sofue \& Handa (1984). The east and west sides of the shell are connected about $1.4^{\circ}$ north of the Galactic plane (not visible in Fig. 1).

A key result of the radio continuum observations is a clear measurement of the $3.6 / 6$ $\mathrm{cm}$ and $6 / 20 \mathrm{~cm}$ radio spectral indices. Figure 2 shows how these spectral indices change with Galactic latitude for the east and west sides of the GCL. The values are generally nonthermal and steepen away from the plane. The similarity in the values for the two sides shows that they are very likely created by similar phenomena. The contiguous change in spectral index and shape also connects the GCL to the nonthermal filament known as the Radio Arc (Yusef-Zadeh, et al. 1984), an object known to be in the central few hundred parsecs of our Galaxy.

\subsection{GBT and Hat Creek Radio Observatory recombination lines near $6 \mathrm{~cm}$}

The ionized gas structure was studied with two separate observations of radio recombination lines (HCRO data courtesy D. Backer). The observations find a shell-like morphology that is similar to radio continuum and mid-IR shells. The GBT observations show that the density and temperature of the ionized gas is similar throughout the GCL. The 


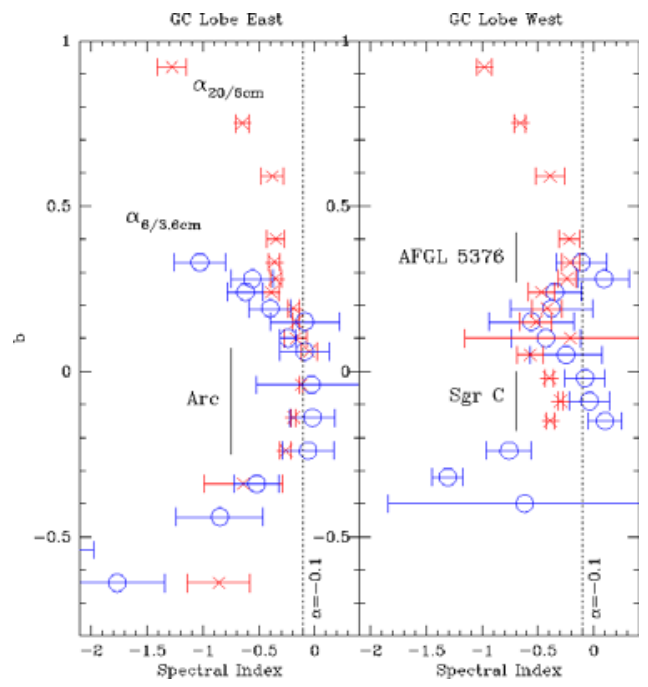

Figure 2. The plots show the dependence of the $6 / 20 \mathrm{~cm}$ and $3.6 / 6 \mathrm{~cm}$ spectral indices on Galactic latitude across the GCL. The left and right panel show the east and west sides of the GCL, respectively.

electron temperature in the ionized gas is unusually low, indicating a high metallicity, which is consistent with the enriched medium in the GC region.

\subsection{SHASSA Ho image}

A different view of the ionized gas is provided by the SHASSA H $\alpha$ survey (Gaustad et al. 2001). Figure 3 shows a combined view of that survey with the HCRO radio recombination line survey, which covers lower latitudes. These maps show that there is a ionized shell of gas that matches the radio continuum and mid-IR shells over the entire structure.

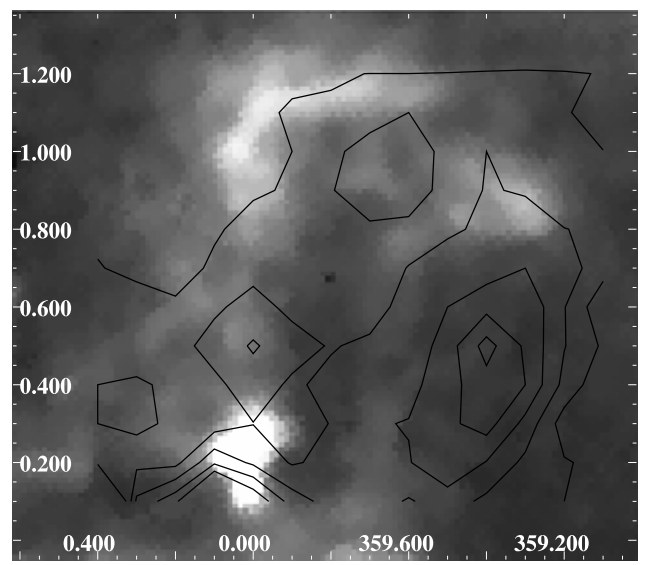

Figure 3. Two surveys of the ionized gas in the GCL. The gray scale shows H $\alpha$ emission (SHASSA; Gaustad et al. 2001) and contours show $6 \mathrm{~cm}$ radio recombination line intensity observed by the HCRO (D. Backer, private communication). The two surveys show ionized gas, but are both biased differently by absorption and incomplete coverage. When combined, they provide a more complete view of the ionized gas in the GCL. 


\subsection{Spitzer/IRAC $8 \mu$ m images}

The GCL was surveyed in part by a Spitzer survey of the GC region (Stolovy et al. 2008 "in prep.") and the GLIMPSE survey (Benjamin et al. 2005). These surveys show that the mid-IR emission at arcsecond resolution traces a complete shell, much like that seen in radio continuum and recombination lines. This emission is dominated by PAH emission, which is caused by irradiation of dust.

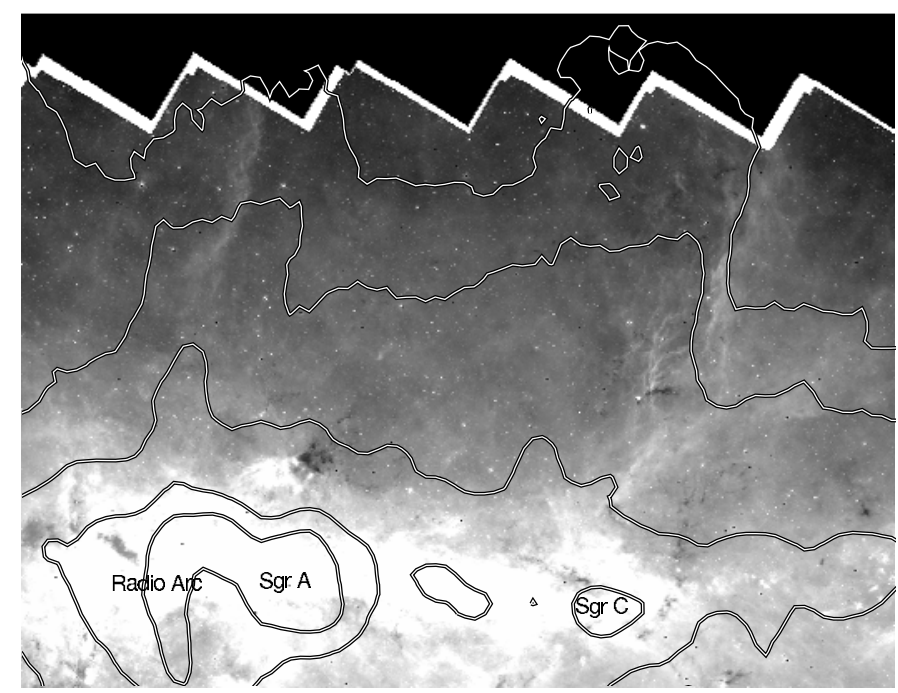

Figure 4. The $8 \mu \mathrm{m}$ emission in the region of the GCL, as observed by Spitzer (from Stolovy et al. 2008).

\subsection{Nature of $G C L$}

The observations show that the GCL is composed of nested shells of gas and dust. There are three, morphologically-distinct components to the GCL, tracing synchrotronemitting, magnetized gas (radio continuum), ionized gas (radio recombination and $\mathrm{H} \alpha$ lines), and PAH-emitting dust ( $8 \mu \mathrm{m}$ continuum). Figure 5 shows how these three components align relative to each other.

The full analysis leads us to a few basic conclusions about the GCL:

- The GCL is a single object: The distinct morphology of the shell and the connection of the east and west sides at the top of the shell argues that the GCL has a single origin.

- The GCL is composed of layered shells: The multiple components are clearly distinct at arcminute size scales.

- The GCL is in the GC region: The connection of the GCL to the Radio Arc (YusefZadeh et al. 1984) and the low electron temperature argue that the entire structure is in the central few hundred parsecs of the Galaxy.

Assuming the GCL is in the GC region (at a distance of roughly $8 \mathrm{kpc}$ ), the pressure measured by thermal x-rays, radio recombination line, and others means implies a formation energy $E \approx 4 \times 10^{52}$ ergs, assuming adiabatic expansion. Upper limits on the expansion velocity from radio recombination line observations show that the shell is not expanding along the line of sight faster than roughly $10 \mathrm{~km} \mathrm{~s}^{-1}$. 


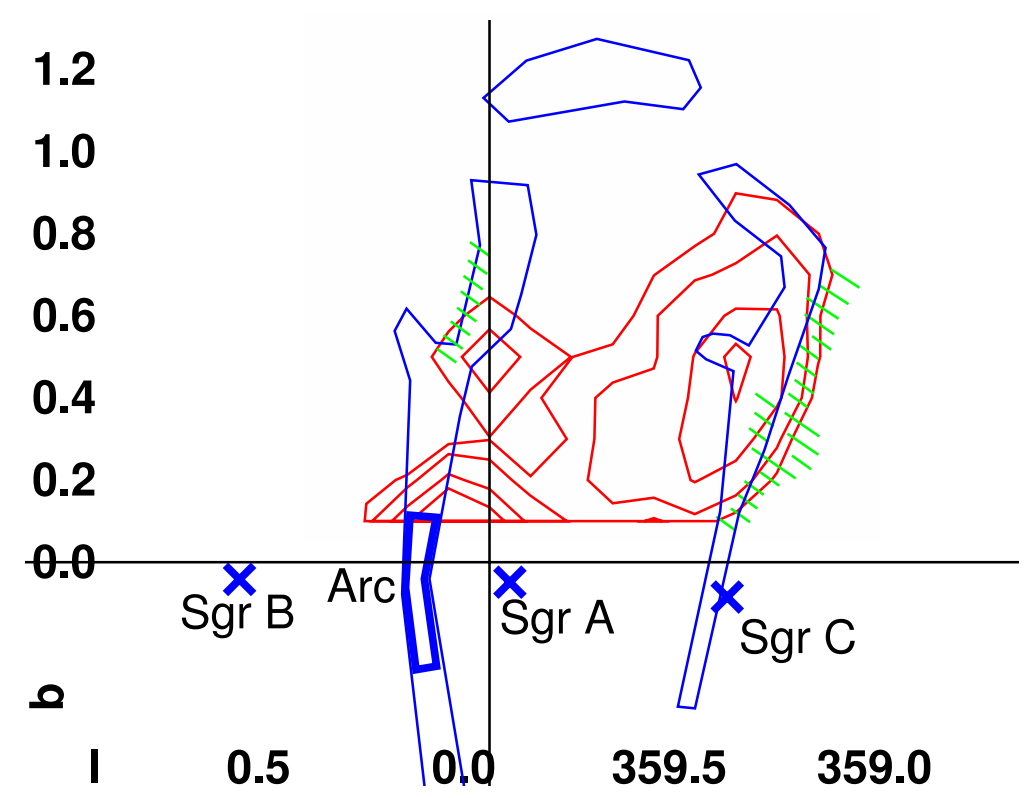

Figure 5. A schematic view of the morphology of the GCL. Red contours show radio recombination line emission. Blue line schematically shows the radio continuum emission. The green hatching schematically shows where the $8 \mu \mathrm{m}$ emission lies.

\section{Formation Models for the GCL}

The physical structure of the GCL suggests that it is an outflow from the center of the Galaxy. Starting with this assumption, we can test models for its formation. The four standard models presented for the formation of the GCL are:

- A small-scale starburst (Chevalier 1992, Bland-Hawthorn \& Cohen 2003) Stellar winds and supernovae create a hot, buoyant bubble of gas that rises from the disk.

- A jet from Sgr A* (Melia \& Falcke 2001) - Jets from accreting massive black holes are often found to create mass outflows.

- Escape of $8 \mathrm{keV}$ gas (Muno et al. 2004, Belmont et al. 2005) — The GC region is believed to be filled with a hot gas of mysterious origin that may buoyantly escape.

- A magnetodynamic effect (Uchida et al. 1985, Shibata \& Uchida 1987) — The inward migration of gas may twist the GC magnetosphere, possibly forcing ionized gas away from the plane.

We find that the morphology and physical conditions in the GCL are most consistent with the starburst model. The observed supernova rate in the central $20 \mathrm{pc}\left(\sim 10^{-5}\right.$ $\mathrm{yr}^{-1}$; Muno et al 2004) could power the GCL after $10^{7} \mathrm{yr}$ (for $\chi \approx 0.2$ ). The GCL is similar to the outflow seen in dwarf galaxy IC 10, which has layered nonthermal and thermal shells with no apparent expansion (Thurow \& Wilcots 2005). In general, the formation energy $\left(\sim 4 \times 10^{52}\right.$ ergs $)$ and ionized mass $\left(3 \times 10^{5} \mathrm{M}_{\odot}\right)$ are similar that seen in dwarf starbursts (Veilleux et al. 2005). Furthermore, the upper limit on the expansion of the GCL is consistent with predictions of scaling relations, given the moderate star formation rate in the GC region and the GCL's small size (Martin 2005). 


\section{Conclusions}

This work has presented new observations that show that the Galactic center lobe is a layered, shell-like structure in our Galactic center. The energy required to form it is consistent with the current supernova rate in the GC region, which suggests that it could be formed by the current star formation there. If so, the small size of the GCL and moderate star formation rate that powers it suggest that this kind of outflow is common. Furthermore, such an outflow would not easily be detected in the nearest spiral galaxies. Thus, the proximity of the GCL provides a unique perspective of this exciting phenomenon.

\section{References}

Belmont, R., Tagger, M., Muno, M. et al. 2005, ApJ, 631, L53

Benjamin, R. A., Churchwell, E., Babler, B. L. et al. 2005, ApJ, 630, L149

Bland-Hawthorn, J. \& Cohen, M. 2003, ApJ, 582, 246

Cecil, G., Bland-Hawthorn, J., Veilleux, S. et al. 2001, ApJ, 555, 338

Chevalier, R. A. 1992, ApJ, 397, L39

Eckart, A. \& Genzel, R. 1996, Nature, 383, 415

Figer, D. F., McLean, I. S., \& Morris, M. 1999, ApJ, 514, 202

Figer, D. F., Rich, R. M., Kim, S. S. et al. 2004, ApJ, 601, 319

Gaustad, J. E., McCullough, P. R., Rosing, W., \& Van Buren, D. 2001, PASP, 113, 1326

Ghez, A., Salim, S., Hornstein, S. D. et al. 2005, ApJ, 620, 744

Law, C. J., et al. 2008, in preparation

Martin, C. L., 2005 ApJ, 621, 227

Melia, F. \& Falcke, H., 2001 ARA\& $\&$, 39, 309

Muno, M. P. Baganoff, F. K., \& Bautz, M. W., 2004 ApJ, 613, 326

Rodríguez-Fernández, N. J. \& Martín-Pintado, J., 2005 A $\& A$, 429, 923

Shibata, K. \& Uchida, Y., 1987 PASJ, 39, 559

Sofue, Y. \& Handa, T., 1984 Nature, 310, 568

Thurow, J. C. \& Wilcots, E. M., 2005 AJ, 129, 745

Uchida, K. I., Morris, M. R., Serabyn, E. \& Bally, J., 1994 ApJ, 421, 505

Uchida, Y., Sofue, Y. \& Shibata, K., 1985 Nature, 317, 699

Veilleux, S., Cecil, G., \& Bland-Hawthorn, J., 2005 ARA\&A, 43, 769

Yusef-Zadeh, F., Morris, M., \& Chance, D., 1984 Nature, 310, 557 